\title{
Student Perception on the Learning Service at Faculty of Science and Technology of Universitas Terbuka
}

\author{
Lintang Patria*, Heny Kurniawati, Nurmala Pangaribuan and Tutisiana Silawati \\ Departement of Information System, Universitas Terbuka, Tangerang Selatan, Indonesia \\ *Corresponding author. Email: lintang@ecampus.ut.ac.id
}

\begin{abstract}
This study intending knows the student perception of learning services in the FST UT. Some of the services that will be this research topic are General Services, Registration Services, Academic Guidance Services, Tutorial Services, Practical Services/Practicum/Studio, Teaching Materials Services, Exam Services and Credit Transfer Services. The output of this research recommendations for service improvement and service innovation at UT FST based on student needs. With services that focus on student satisfaction, it is expected that UT FST students can learning well and then successfully graduate on time. Based on this study, it was found that $43 \%$ of students were very satisfied, $38 \%$ said they were satisfied, $10 \%$ said they were quite satisfied, and 1\% felt dissatisfied. $7 \%$ of students did not answer questions, especially in studio practicum services, teaching materials services and credit transfer services. Important information that students want is about General Services, Tutorial Services, Teaching Materials Services, Practicum Services and Exam Services. Based on this research, the service that needs to be developed is a service through WhatsApp group (because with this service development, problems in service to students can be canceled).
\end{abstract}

Keywords: service, academic advisor, FST

\section{INTRODUCTION}

Universitas Terbuka (UT/Open University) was inaugurated in 1984 through Presidential Decree No.41 of 1984. UT was designed to be a university with a LongDistance Education (PJJ) system. Through PJJ, UT is here to facilitate all Indonesian citizens to get the widest possible opportunity to study in higher education without being constrained by age, geographic location, and demography, including economic factors. In participating in learning at UT, students get registration services, tutorial services, teaching materials services, examination services. Registration services are carried out through the UT website or come directly to the nearest UPBJJ. The tutorial service is done face-to-face or online. Practicum services are carried out for study programs that require practicum in their curriculum. To get the service of teaching materials, students must order the book at The Online Book Shop (TBO) Service written exam conducted at a predetermined test or through Online Exams (UO).

The research "Student Perception of the Service Learning at FST UT “ wants to find how students' perception on the learning services offered by the UT, especially for students FST UT. This research aimed to obtain information related to the Public Service, Registration, Academic Guidance, Tutorial, Practicum/ Lab/ Studio, Instructional Materials, Exam, Credit Transfer as well as a learning experience at UT [1]-[2].

The benefits of this research are to identify student perception towards service learning in academic and administrative FST UT. Furthermore, it can be used as input for management, to improve services for UT students in the academic and administrative unit.

\section{LITERATURE REVIEW}

Higher education aims to prepare students to become members of society who have academic and professional abilities that can apply, develop and create knowledge, technology and arts as well as develop and disseminate the knowledge. To achieve these goals, Universities are required to carry out learning and create a conducive atmosphere, complete and adequate facilities and infrastructure to provide integrated guidance [3]-[5]. 
Quality of academic services in university is an activity of providing services in the form of meeting all academic needs that are able to meet or exceed student expectations as customers in higher education [6]-[12].

The development of Long-Distance learning in the last decade has grown very rapidly, more and more universities are opening programs with a distance education system, which makes prospective students have many options to choose the college they are interested in. Therefore, the competition between colleges that offer distance education is very high. This forces LongDistance learning organizers to improve academic and administrative services; thus, user / student satisfaction is fulfilled. Student's satisfaction during the distance education system is taken into account because this case affects the diligence of students attending lectures, especially in the FST UT. Information about students perception on learning services in UT FST would be useful for the evaluation of FST services and to develop better services of FST academic. Through this research, it is expected that all citizens of FST can expressed themselves and pulled forward Education in Indonesia.

\section{RESEARCH METHODOLOGY}

The research procedure is how the researcher performs his job in designing the research. The final objective of this research is to know the responses, aspirations and expectations of students towards FST services. Based on these objectives, the researchers designed the research design, population and sample determination, planning methods to be used and analysis of the questionnaire results.

\subsection{Research Design}

Respondents of this study consisted of active FST students in the 2019/20.2 and 2020/21.1 periods. The instrument is a questionnaire. Researchers sent a message through WhatsApp to students to fill out a questionnaire online. The research was conducted using a quantitative method because the limited interviews in Pandemic of COVID-19.

\subsection{Population and Sample}

The population of this research is the FST active students during the 2019 / 20.2 and 2020 / 21.1. The samples of this study were students from several UPBJJ-UT. The results are identified through Simple Random System. The researcher sent WA and email to all FST students.

\subsection{Data Collecting Method}

Primary data were obtained through a survey using questionnaire by WA or email to each respondent. Secondary data obtained and collected from various literature, books, journals, theses, dissertations and data from the Internet that are considered relevant.
Measurement data will be conducted using Likert Scale using the value as follows : $1=$ not satisfied, $2=$ fairly satisfied, $3=$ satisfied, $4=$ very satisfied.

\subsection{Research Instrument}

The research instrument refers to the purpose of the study, the instruments for the students divided into 9 sections which are: (1) General Service, (2) Registration Service, (3) Academic Consultation Service, (4) Tutorial Service, (5) Practicum/ Laboratory/ Studio Service, (6) Learning Material Service, (7) Exam Service, (8) Transfer Credit Service, and (9) Learning Experience in UT.

\subsection{Data Collection and Analysis Technique}

The data was collected only through online questionnaire. The results of the input from students were analyzed descriptively.

\section{RESULTS AND DISCUSSION}

\subsection{Respondents Overview}

This research was conducted by distributing online questionnaires using Google Form by sending it through WhatsApp and E-mail blast method to students. The contents of the messages are an appeal to fill out the questionnaire.

The number of respondents who filled out the questionnaire was 258 with a composition of $53 \%$ male and $47 \%$ female. The respondents are scattered in 37 UPBJJ. The respondent come from 7 undergraduate program namely: Agribusiness, Agriculture, Agribusiness Fisheries, Agribusiness Husbandry, Mathematics, Statistics, Urban and Regional Planning, Food Science Technology, Information Systems, and 1 graduate program. The year of registration of respondents was spread from 2002 to 2020 .

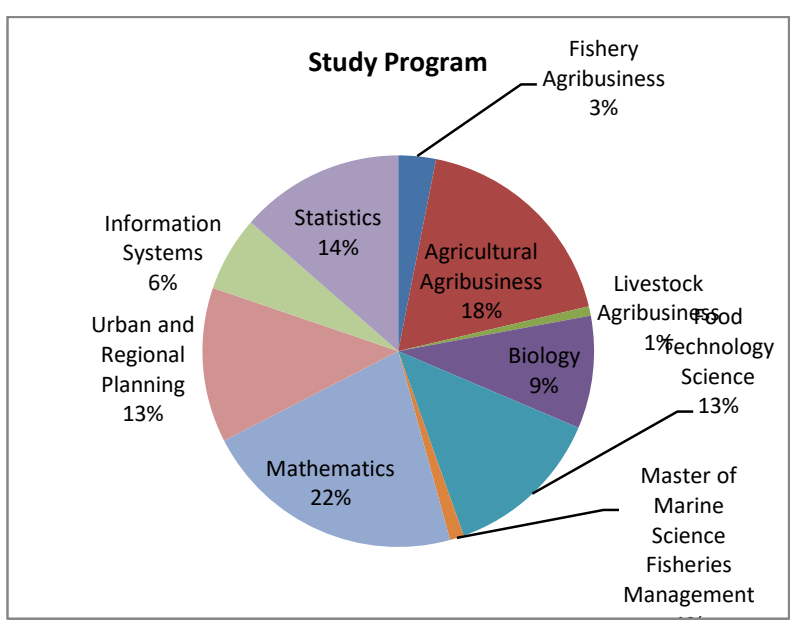

Figure 1 Percentage Distribution of Students' Program 


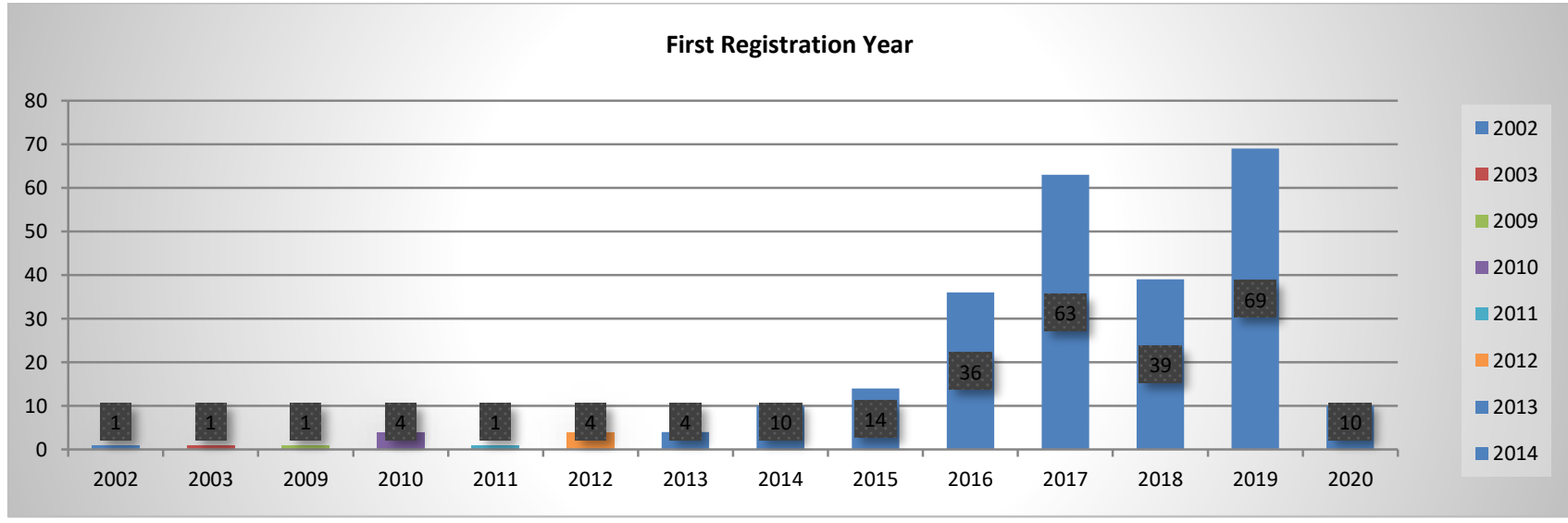

Figure 2. Distribution of Student's Year of Registration

Table 1 Student Perceptions of General Service

\begin{tabular}{|c|c|c|c|c|c|}
\hline \multirow{2}{*}{ A. GENERAL SERVICE } & \multicolumn{5}{|c|}{ PERCENTAGE } \\
\hline & 1 & 2 & 3 & 4 & Not Answered \\
\hline \multicolumn{6}{|l|}{ 1. Clarity of information about UT } \\
\hline Satisfaction & $1 \%$ & $8 \%$ & $48 \%$ & $43 \%$ & $0 \%$ \\
\hline Interest & $0 \%$ & $5 \%$ & $45 \%$ & $50 \%$ & $0 \%$ \\
\hline \multicolumn{6}{|c|}{$\begin{array}{l}\text { 2. Tuition fees paid by students compared to the } \\
\text { services provided }\end{array}$} \\
\hline Satisfaction & $1 \%$ & $11 \%$ & $36 \%$ & $52 \%$ & $0 \%$ \\
\hline Interest & $0 \%$ & $7 \%$ & $40 \%$ & $53 \%$ & $0 \%$ \\
\hline \multicolumn{6}{|l|}{ 3. Ease of contacting UT Staff } \\
\hline Satisfaction & $2 \%$ & $24 \%$ & $41 \%$ & $32 \%$ & $0 \%$ \\
\hline Interest & $1 \%$ & $15 \%$ & $38 \%$ & $47 \%$ & $0 \%$ \\
\hline \multicolumn{6}{|l|}{ 4. Ease of contacting the Tutor } \\
\hline Satisfaction & $5 \%$ & $24 \%$ & $47 \%$ & $24 \%$ & $0 \%$ \\
\hline Interest & $0 \%$ & $15 \%$ & $41 \%$ & $43 \%$ & $0 \%$ \\
\hline \multicolumn{6}{|l|}{ 5. Hospitality of UT staff in serving students } \\
\hline Satisfaction & $2 \%$ & $12 \%$ & $38 \%$ & $48 \%$ & $0 \%$ \\
\hline Interest & $1 \%$ & $10 \%$ & $32 \%$ & $57 \%$ & $0 \%$ \\
\hline \multicolumn{6}{|c|}{ 6. Speed of complaint handling / case handling } \\
\hline Satisfaction & $3 \%$ & $21 \%$ & $49 \%$ & $27 \%$ & $0 \%$ \\
\hline Interest & $2 \%$ & $15 \%$ & $38 \%$ & $46 \%$ & $0 \%$ \\
\hline \multicolumn{6}{|l|}{ 7. Credit transfer service } \\
\hline Satisfaction & $3 \%$ & $22 \%$ & $49 \%$ & $25 \%$ & $0 \%$ \\
\hline Interest & $3 \%$ & $18 \%$ & $43 \%$ & $36 \%$ & $0 \%$ \\
\hline \multicolumn{6}{|l|}{ 8.Exam Service } \\
\hline Satisfaction & $0 \%$ & $8 \%$ & $43 \%$ & $48 \%$ & $0 \%$ \\
\hline Interest & $0 \%$ & $6 \%$ & $36 \%$ & $59 \%$ & $0 \%$ \\
\hline
\end{tabular}


Table 2 Student Perceptions of Registration Service

\begin{tabular}{lccccc}
\hline \multicolumn{1}{c}{ B. REGISTRATION SERVICE } & \multicolumn{5}{c}{ PERCENTAGE } \\
\cline { 2 - 6 } & 1 & 2 & 3 & 4 & Not Answered \\
\hline 9. Registration file processing service & & & & & $0 \%$ \\
Satisfaction & $0 \%$ & $7 \%$ & $32 \%$ & $62 \%$ & $0 \%$ \\
Interest & $0 \%$ & $4 \%$ & $30 \%$ & $66 \%$ & $0 \%$ \\
10. Registration case resolution services & & & & & $0 \%$ \\
Satisfaction & $1 \%$ & $7 \%$ & $45 \%$ & $46 \%$ & $55 \%$ \\
Interest & $0 \%$ & $5 \%$ & $39 \%$ & $5 \%$ & \\
\hline
\end{tabular}

Students' perceptions of the Registration service were quite good, it can be seen from the presentation of students that answered "very satisfied"

Table 3 Student Perceptions of Academic Consultation Service

\begin{tabular}{|c|c|c|c|c|c|}
\hline \multirow{2}{*}{ C. ACADEMIC CONSULTATION SERVICE } & \multicolumn{5}{|c|}{ PERCENTAGE } \\
\hline & 1 & 2 & 3 & 4 & Not Answered \\
\hline \multicolumn{6}{|l|}{ 12. Guidance for Course Registration } \\
\hline Satisfaction & $1 \%$ & $12 \%$ & $44 \%$ & $43 \%$ & $0 \%$ \\
\hline Interest & $0 \%$ & $8 \%$ & $40 \%$ & $53 \%$ & $0 \%$ \\
\hline \multicolumn{6}{|l|}{ 13. Guidance if there are academic problems } \\
\hline Satisfaction & $1 \%$ & $17 \%$ & $51 \%$ & $31 \%$ & $0 \%$ \\
\hline Interest & $0 \%$ & $14 \%$ & $40 \%$ & $46 \%$ & $0 \%$ \\
\hline \multicolumn{6}{|l|}{ 14. Credit transfer guidance } \\
\hline Satisfaction & $3 \%$ & $26 \%$ & $48 \%$ & $23 \%$ & $0 \%$ \\
\hline Interest & $3 \%$ & $18 \%$ & $44 \%$ & $35 \%$ & $0 \%$ \\
\hline \multicolumn{6}{|l|}{ 15. Guidance for dealing with Final Exam } \\
\hline Satisfaction & $3 \%$ & $20 \%$ & $45 \%$ & $33 \%$ & $0 \%$ \\
\hline Interest & $2 \%$ & $14 \%$ & $37 \%$ & $48 \%$ & $0 \%$ \\
\hline \multicolumn{6}{|l|}{ 16. Guidance for Practicum / Study } \\
\hline Satisfaction & $4 \%$ & $16 \%$ & $45 \%$ & $18 \%$ & $9 \%$ \\
\hline Interest & $2 \%$ & $12 \%$ & $38 \%$ & $40 \%$ & $9 \%$ \\
\hline \multicolumn{6}{|l|}{ 17. Guidance for final task/ theses } \\
\hline Satisfaction & $2 \%$ & $17 \%$ & $45 \%$ & $25 \%$ & $11 \%$ \\
\hline Interest & $1 \%$ & $8 \%$ & $39 \%$ & $40 \%$ & $12 \%$ \\
\hline \multicolumn{6}{|l|}{$\begin{array}{l}\text { 18.Communication with UT Lecturers (Central and } \\
\text { UPBJJ) }\end{array}$} \\
\hline Satisfaction & $3 \%$ & $17 \%$ & $48 \%$ & $32 \%$ & $0 \%$ \\
\hline Interest & $2 \%$ & $11 \%$ & $38 \%$ & $50 \%$ & $0 \%$ \\
\hline \multicolumn{6}{|l|}{$\begin{array}{l}\text { 19. Direct service with UT lecturers (Central and } \\
\text { UPBJJ) }\end{array}$} \\
\hline Satisfaction & $3 \%$ & $15 \%$ & $49 \%$ & $33 \%$ & $0 \%$ \\
\hline Interest & $2 \%$ & $10 \%$ & $40 \%$ & $48 \%$ & $0 \%$ \\
\hline
\end{tabular}


Academic Guidance Service needs to be improved; it can be seen from the students' answer that only "satisfied".

Table 4 Student Perceptions of the Tutorial service

\begin{tabular}{|c|c|c|c|c|c|}
\hline \multirow{2}{*}{ D. TUTORIAL SERVICES } & \multicolumn{5}{|c|}{ PERCENTAGE } \\
\hline & 1 & 2 & 3 & 4 & Not Answered \\
\hline \multicolumn{6}{|l|}{$\begin{array}{l}\text { 20. Registration Procedure of Face-to-Face } \\
\text { Tutorial/ Tuton }\end{array}$} \\
\hline Satisfaction & $1 \%$ & $5 \%$ & $35 \%$ & $58 \%$ & $0 \%$ \\
\hline Interest & $0 \%$ & $3 \%$ & $31 \%$ & $65 \%$ & $0 \%$ \\
\hline \multicolumn{6}{|l|}{ 21. Tutors Mastery } \\
\hline Satisfaction & $1 \%$ & $6 \%$ & $55 \%$ & $38 \%$ & $0 \%$ \\
\hline Interest & $0 \%$ & $7 \%$ & $38 \%$ & $55 \%$ & $0 \%$ \\
\hline \multicolumn{6}{|l|}{$\begin{array}{l}\text { 22. The role of the tutor in helping students } \\
\text { understand course material }\end{array}$} \\
\hline Satisfaction & $1 \%$ & $17 \%$ & $50 \%$ & $32 \%$ & $0 \%$ \\
\hline Interest & $0 \%$ & $11 \%$ & $38 \%$ & $51 \%$ & $0 \%$ \\
\hline \multicolumn{6}{|c|}{$\begin{array}{l}\text { 23. Feedback provided by tutors on exercises / } \\
\text { assignments }\end{array}$} \\
\hline Satisfaction & $2 \%$ & $14 \%$ & $53 \%$ & $31 \%$ & $0 \%$ \\
\hline Interest & $0 \%$ & $7 \%$ & $41 \%$ & $51 \%$ & $0 \%$ \\
\hline \multicolumn{6}{|c|}{$\begin{array}{l}\text { 24. Suitability of the tutorial implementation with } \\
\text { the schedule }\end{array}$} \\
\hline Satisfaction & $0 \%$ & $6 \%$ & $43 \%$ & $51 \%$ & $0 \%$ \\
\hline Interest & $0 \%$ & $2 \%$ & $39 \%$ & $59 \%$ & $0 \%$ \\
\hline \multicolumn{6}{|l|}{ 25. Tutorial Assessment } \\
\hline Satisfaction & $2 \%$ & $11 \%$ & $50 \%$ & $37 \%$ & $0 \%$ \\
\hline Interest & $1 \%$ & $8 \%$ & $38 \%$ & $53 \%$ & $0 \%$ \\
\hline \multicolumn{6}{|c|}{$\begin{array}{l}\text { 26. The suitability of the tutorial material with Final } \\
\text { Exam questions }\end{array}$} \\
\hline Satisfaction & $2 \%$ & $21 \%$ & $49 \%$ & $27 \%$ & $0 \%$ \\
\hline Interest & $2 \%$ & $10 \%$ & $39 \%$ & $49 \%$ & $0 \%$ \\
\hline \multicolumn{6}{|l|}{ 27. Communication with tutors } \\
\hline Satisfaction & $3 \%$ & $21 \%$ & $49 \%$ & $27 \%$ & $0 \%$ \\
\hline Interest & $1 \%$ & $12 \%$ & $43 \%$ & $45 \%$ & $0 \%$ \\
\hline \multicolumn{6}{|l|}{ 28. Ease of access to the tutorial location } \\
\hline Satisfaction & $2 \%$ & $14 \%$ & $42 \%$ & $33 \%$ & $9 \%$ \\
\hline Interest & $1 \%$ & $11 \%$ & $35 \%$ & $43 \%$ & $10 \%$ \\
\hline \multicolumn{6}{|l|}{ 29. Ease of access to tuton } \\
\hline Satisfaction & $0 \%$ & $8 \%$ & $34 \%$ & $55 \%$ & $3 \%$ \\
\hline Interest & $0 \%$ & $4 \%$ & $31 \%$ & $62 \%$ & $3 \%$ \\
\hline
\end{tabular}

In general, students are satisfied with the Tutorial service, and we must maintain service and provide service innovation so that students become more satisfied. 
Table 5 Student Perceptions of Practicum / Lab./ Studio services

\begin{tabular}{|c|c|c|c|c|c|}
\hline \multirow[t]{2}{*}{ E. PRACTICUM/ LAB./ STUDIO SERVICES } & \multicolumn{5}{|c|}{ PERCENTAGE } \\
\hline & 1 & 2 & 3 & 4 & Not Answered \\
\hline \multicolumn{6}{|l|}{$\begin{array}{l}\text { 30. Socialization of the time for practicum/ Lab./ } \\
\text { Studio }\end{array}$} \\
\hline Satisfaction & $1 \%$ & $18 \%$ & $39 \%$ & $23 \%$ & $19 \%$ \\
\hline Interest & $1 \%$ & $12 \%$ & $35 \%$ & $34 \%$ & $19 \%$ \\
\hline \multicolumn{6}{|l|}{$\begin{array}{l}\text { 31. Ease of obtaining a schedule for the practicum/ } \\
\text { Lab./ Studio }\end{array}$} \\
\hline Satisfaction & $2 \%$ & $16 \%$ & $41 \%$ & $23 \%$ & $19 \%$ \\
\hline Interest & $2 \%$ & $9 \%$ & $34 \%$ & $38 \%$ & $19 \%$ \\
\hline \multicolumn{6}{|l|}{$\begin{array}{l}\text { 32. Suitability of the time of practicum/ Lab./ } \\
\text { Studio with the schedule }\end{array}$} \\
\hline Satisfaction & $0 \%$ & $14 \%$ & $43 \%$ & $24 \%$ & $19 \%$ \\
\hline Interest & $0 \%$ & $10 \%$ & $36 \%$ & $36 \%$ & $19 \%$ \\
\hline \multicolumn{6}{|l|}{ 33. Mastery of Instructor Material } \\
\hline Satisfaction & $0 \%$ & $9 \%$ & $48 \%$ & $25 \%$ & $18 \%$ \\
\hline Interest & $0 \%$ & $6 \%$ & $41 \%$ & $35 \%$ & $18 \%$ \\
\hline \multicolumn{6}{|l|}{$\begin{array}{l}\text { 34. Instructor's role in assisting the implementation } \\
\text { of practicum/ Lab./ Studio }\end{array}$} \\
\hline Satisfaction & $0 \%$ & $11 \%$ & $43 \%$ & $28 \%$ & $18 \%$ \\
\hline Interest & $0 \%$ & $6 \%$ & $40 \%$ & $36 \%$ & $19 \%$ \\
\hline \multicolumn{6}{|l|}{$\begin{array}{l}\text { 35. Feedback given by the instructor during the } \\
\text { implementation of practicum/ Lab./ Studio }\end{array}$} \\
\hline Satisfaction & $0 \%$ & $14 \%$ & $41 \%$ & $27 \%$ & $19 \%$ \\
\hline Interest & $0 \%$ & $9 \%$ & $40 \%$ & $32 \%$ & $19 \%$ \\
\hline \multicolumn{6}{|l|}{$\begin{array}{l}\text { 36. Completeness of equipment for practicum/ } \\
\text { Lab./ Studio }\end{array}$} \\
\hline Satisfaction & $1 \%$ & $14 \%$ & $45 \%$ & $21 \%$ & $19 \%$ \\
\hline Interest & $0 \%$ & $10 \%$ & $40 \%$ & $30 \%$ & $19 \%$ \\
\hline \multicolumn{6}{|l|}{ 37. Assessment of practicum/ Lab./ Studio } \\
\hline Satisfaction & $0 \%$ & $12 \%$ & $45 \%$ & $24 \%$ & $19 \%$ \\
\hline Interest & $0 \%$ & $9 \%$ & $40 \%$ & $32 \%$ & $19 \%$ \\
\hline
\end{tabular}

In general, students' perceptions of Practicum, Laboratory and Studio services are still in the "satisfied" category, so we need to improve these services.

Table 6 Students' Perceptions of Teaching Materials

\begin{tabular}{|c|c|c|c|c|c|}
\hline \multirow{2}{*}{ F. TEACHING MATERIALS } & \multicolumn{5}{|c|}{ PERCENTAGE } \\
\hline & 1 & 2 & 3 & 4 & Not Answered \\
\hline \multicolumn{6}{|l|}{ 38. Ease of obtaining teaching materials } \\
\hline Satisfaction & $2 \%$ & $10 \%$ & $71 \%$ & $56 \%$ & $0 \%$ \\
\hline Interest & $1 \%$ & $5 \%$ & $25 \%$ & $69 \%$ & $0 \%$ \\
\hline \multicolumn{6}{|l|}{ 39. The speed of receiving teaching materials } \\
\hline Satisfaction & $2 \%$ & $13 \%$ & $47 \%$ & $38 \%$ & $0 \%$ \\
\hline
\end{tabular}




\begin{tabular}{|c|c|c|c|c|c|}
\hline \multirow{2}{*}{ F. TEACHING MATERIALS } & \multicolumn{5}{|c|}{ PERCENTAGE } \\
\hline & 1 & 2 & 3 & 4 & Not Answered \\
\hline Interest & $2 \%$ & $7 \%$ & $32 \%$ & $59 \%$ & $0 \%$ \\
\hline \multicolumn{6}{|l|}{ 40. Ease of understanding teaching materials } \\
\hline Satisfaction & $4 \%$ & $18 \%$ & $55 \%$ & $23 \%$ & $0 \%$ \\
\hline Interest & $1 \%$ & $12 \%$ & $37 \%$ & $50 \%$ & $0 \%$ \\
\hline \multicolumn{6}{|l|}{$\begin{array}{l}\text { 41. Quality of physical packaging of teaching } \\
\text { materials }\end{array}$} \\
\hline Satisfaction & $3 \%$ & $7 \%$ & $37 \%$ & $53 \%$ & $0 \%$ \\
\hline Interest & $1 \%$ & $3 \%$ & $38 \%$ & $57 \%$ & $0 \%$ \\
\hline \multicolumn{6}{|l|}{ 42. Quality of the material } \\
\hline Satisfaction & $2 \%$ & $10 \%$ & $46 \%$ & $43 \%$ & $0 \%$ \\
\hline Interest & $1 \%$ & $5 \%$ & $34 \%$ & $60 \%$ & $0 \%$ \\
\hline $\begin{array}{l}\text { Numbers } 43,44 \text { and } 45 \text { are filled if you order } \\
\text { teaching materials through the Online Bookstore } \\
\text { (TBO) }\end{array}$ & \multicolumn{5}{|c|}{ PRESENTASE } \\
\hline \multicolumn{6}{|l|}{ 43. Ease of use of the TBO application } \\
\hline Satisfaction & $1 \%$ & $7 \%$ & $24 \%$ & $27 \%$ & $42 \%$ \\
\hline Interest & $1 \%$ & $4 \%$ & $20 \%$ & $34 \%$ & $41 \%$ \\
\hline \multicolumn{6}{|l|}{ 44. Availability of teaching materials at TBO } \\
\hline Satisfaction & $0 \%$ & $6 \%$ & $26 \%$ & $27 \%$ & $41 \%$ \\
\hline Interest & $1 \%$ & $3 \%$ & $18 \%$ & $36 \%$ & $42 \%$ \\
\hline \multicolumn{6}{|l|}{$\begin{array}{l}\text { 45. The conformity of teaching materials received } \\
\text { and those ordered }\end{array}$} \\
\hline Satisfaction & $1 \%$ & $4 \%$ & $18 \%$ & $37 \%$ & $40 \%$ \\
\hline Interest & $1 \%$ & $2 \%$ & $15 \%$ & $42 \%$ & $40 \%$ \\
\hline \multirow[t]{2}{*}{$\begin{array}{c}\text { Number } 46 \text { is only filled if you follow the SIPAS } \\
\text { program with face-to-face tutorial }\end{array}$} & \multicolumn{5}{|c|}{ PRESENTASE } \\
\hline & 1 & 2 & 3 & 4 & Not Answered \\
\hline \multicolumn{6}{|l|}{$\begin{array}{l}\text { 46. Teaching materials received before the first } \\
\text { tutorial meeting }\end{array}$} \\
\hline Satisfaction & $0 \%$ & $4 \%$ & $13 \%$ & $21 \%$ & $61 \%$ \\
\hline Interest & $1 \%$ & $2 \%$ & $12 \%$ & $24 \%$ & $61 \%$ \\
\hline
\end{tabular}

Most of the students answered "very satisfied", but many did not. This may be because not all students buy teaching materials.

Table 7 Students' Perceptions of the Exam Service

\begin{tabular}{|c|c|c|c|c|c|}
\hline \multirow{2}{*}{ G. EXAM SERVICE } & \multicolumn{5}{|c|}{ PERCENTAGE } \\
\hline & 1 & 2 & 3 & 4 & Not Answered \\
\hline \multicolumn{6}{|l|}{ 47. Exam Schedule } \\
\hline Satisfaction & $0 \%$ & $5 \%$ & $28 \%$ & $66 \%$ & $0 \%$ \\
\hline Interest & $0 \%$ & $2 \%$ & $23 \%$ & $75 \%$ & $0 \%$ \\
\hline \multicolumn{6}{|l|}{ 48. Exam Location } \\
\hline Satisfaction & $1 \%$ & $5 \%$ & $33 \%$ & $61 \%$ & $0 \%$ \\
\hline Interest & $0 \%$ & $3 \%$ & $26 \%$ & $71 \%$ & $0 \%$ \\
\hline
\end{tabular}




\begin{tabular}{|c|c|c|c|c|c|}
\hline \multirow{2}{*}{ G. EXAM SERVICE } & \multicolumn{5}{|c|}{ PERCENTAGE } \\
\hline & 1 & 2 & 3 & 4 & Not Answered \\
\hline \multicolumn{6}{|l|}{ 49. Examination } \\
\hline Satisfaction & $1 \%$ & $5 \%$ & $32 \%$ & $62 \%$ & $0 \%$ \\
\hline Interest & $0 \%$ & $4 \%$ & $25 \%$ & $71 \%$ & $0 \%$ \\
\hline \multicolumn{6}{|l|}{ 50. Assessment System } \\
\hline Satisfaction & $3 \%$ & $21 \%$ & $40 \%$ & $37 \%$ & $0 \%$ \\
\hline Interest & $1 \%$ & $12 \%$ & $32 \%$ & $55 \%$ & $0 \%$ \\
\hline \multicolumn{6}{|c|}{$\begin{array}{l}\text { 51. The accuracy of the result announcement } \\
\text { schedule }\end{array}$} \\
\hline Satisfaction & $2 \%$ & $17 \%$ & $41 \%$ & $40 \%$ & $0 \%$ \\
\hline Interest & $0 \%$ & $7 \%$ & $32 \%$ & $60 \%$ & $0 \%$ \\
\hline \multicolumn{6}{|c|}{$\begin{array}{l}\text { 52. Dissemination of information regarding online } \\
\text { examination system }\end{array}$} \\
\hline Satisfaction & $2 \%$ & $17 \%$ & $49 \%$ & $32 \%$ & $0 \%$ \\
\hline Interest & $2 \%$ & $7 \%$ & $41 \%$ & $50 \%$ & $0 \%$ \\
\hline \multicolumn{6}{|c|}{$\begin{array}{l}\text { 53. Dissemination of information regarding } \\
\text { remedial exam }\end{array}$} \\
\hline Satisfaction & $6 \%$ & $21 \%$ & $47 \%$ & $26 \%$ & $0 \%$ \\
\hline Interest & $3 \%$ & $11 \%$ & $39 \%$ & $47 \%$ & $0 \%$ \\
\hline
\end{tabular}

Most of the students stated that they were very satisfied with the examination service. We must maintain the level of student satisfaction by providing innovative examination services that make it easier for students.

Table 8 Students' Perceptions of Credit Transfer Service

\begin{tabular}{lccccc}
\hline \multirow{2}{*}{\multicolumn{1}{c}{ H. TRANSFER CREDIT SERVICES }} & \multicolumn{5}{c}{ PERCENTAGE } \\
\cline { 2 - 6 } & 1 & 2 & 3 & 4 & Not Answered \\
\hline 54. Clarity of information regarding Credit Transfer & & & & & \\
Satisfaction & $4 \%$ & $21 \%$ & $37 \%$ & $14 \%$ & $24 \%$ \\
Interest & $3 \%$ & $14 \%$ & $33 \%$ & $26 \%$ & $23 \%$ \\
55. Ease of Credit Transfer process & & & & & \\
Satisfaction & $5 \%$ & $19 \%$ & $38 \%$ & $14 \%$ & $24 \%$ \\
Interest & $3 \%$ & $14 \%$ & $34 \%$ & $26 \%$ & $24 \%$ \\
56. Credit Transfer Fee & & & & & \\
Satisfaction & $2 \%$ & $17 \%$ & $38 \%$ & $18 \%$ & $24 \%$ \\
Interest & $2 \%$ & $14 \%$ & $33 \%$ & $27 \%$ & $24 \%$ \\
57. Credit Transfer Processing Time & & & & & \\
Satisfaction & $3 \%$ & $21 \%$ & $37 \%$ & $15 \%$ & $24 \%$ \\
Interest & $2 \%$ & $15 \%$ & $34 \%$ & $24 \%$ & $24 \%$ \\
\hline
\end{tabular}


Most students stated that they were very satisfied with credit transfer services. We must maintain the level of student satisfaction by providing innovative credit transfer services that make it easier for students.

Table 9 Students' Perceptions of Learning Experiences at UT

\begin{tabular}{|c|c|c|c|c|c|}
\hline \multirow{2}{*}{ I. LEARNING EXPERIENCE AT UT } & \multicolumn{5}{|c|}{ PERCENTAGE } \\
\hline & 1 & 2 & 3 & 4 & Not Answered \\
\hline \multicolumn{6}{|l|}{$\begin{array}{l}\text { 58. Learning experiences increase knowledge in the } \\
\text { field of science }\end{array}$} \\
\hline Satisfaction & $0 \%$ & $5 \%$ & $42 \%$ & $52 \%$ & $0 \%$ \\
\hline Interest & $0 \%$ & $3 \%$ & $31 \%$ & $66 \%$ & $0 \%$ \\
\hline \multicolumn{6}{|l|}{$\begin{array}{l}\text { 59. Learning experiences improve communication } \\
\text { skills }\end{array}$} \\
\hline Satisfaction & $0 \%$ & $9 \%$ & $47 \%$ & $43 \%$ & $0 \%$ \\
\hline Interest & $0 \%$ & $5 \%$ & $38 \%$ & $57 \%$ & $0 \%$ \\
\hline \multicolumn{6}{|l|}{ 60. Learning experiences improve writing skills } \\
\hline Satisfaction & $0 \%$ & $11 \%$ & $46 \%$ & $42 \%$ & $0 \%$ \\
\hline Interest & $0 \%$ & $8 \%$ & $37 \%$ & $55 \%$ & $0 \%$ \\
\hline \multicolumn{6}{|l|}{$\begin{array}{l}\text { 61. Learning experiences improve skills using } \\
\text { technology }\end{array}$} \\
\hline Satisfaction & $0 \%$ & $7 \%$ & $40 \%$ & $53 \%$ & $0 \%$ \\
\hline Interest & $0 \%$ & $5 \%$ & $33 \%$ & $63 \%$ & $0 \%$ \\
\hline \multicolumn{6}{|l|}{ 62. Learning experiences increase independence } \\
\hline Satisfaction & $0 \%$ & $5 \%$ & $32 \%$ & $63 \%$ & $0 \%$ \\
\hline Interest & $0 \%$ & $3 \%$ & $27 \%$ & $70 \%$ & $0 \%$ \\
\hline \multicolumn{6}{|l|}{$\begin{array}{l}\text { 63. Learning experiences improve time } \\
\text { management skills }\end{array}$} \\
\hline Satisfaction & $0 \%$ & $5 \%$ & $35 \%$ & $60 \%$ & $0 \%$ \\
\hline Interest & $0 \%$ & $3 \%$ & $28 \%$ & $68 \%$ & $0 \%$ \\
\hline \multicolumn{6}{|l|}{$\begin{array}{l}\text { 64. Learning experiences increase the ability to } \\
\text { present ideas, results or reports }\end{array}$} \\
\hline Satisfaction & $0 \%$ & $6 \%$ & $43 \%$ & $51 \%$ & $0 \%$ \\
\hline Interest & $0 \%$ & $4 \%$ & $32 \%$ & $64 \%$ & $0 \%$ \\
\hline \multicolumn{6}{|l|}{ 65. Learning experiences increase self-confidence } \\
\hline Satisfaction & $1 \%$ & $8 \%$ & $43 \%$ & $48 \%$ & $0 \%$ \\
\hline Interest & $0 \%$ & $4 \%$ & $31 \%$ & $64 \%$ & $0 \%$ \\
\hline
\end{tabular}

Most students stated that they were very satisfied with the learning experience at UT. We must maintain the level of student satisfaction by providing a learning experience that makes it easier for students. In summary, the following are students' views of services at FST UT. 
Table 10 Summary of Student Perceptions of services at FST UT

\begin{tabular}{|c|c|c|c|c|c|}
\hline Type of Service & Not satisfied & Quite satisfied & Satisfied & $\begin{array}{l}\text { Very } \\
\text { satisfied }\end{array}$ & $\begin{array}{l}\text { Not } \\
\text { Answered }\end{array}$ \\
\hline A. GENERAL SERVICES & $2 \%$ & $14 \%$ & $42 \%$ & $43 \%$ & $0 \%$ \\
\hline B. REGISTRATION SERVICES & $2 \%$ & $14 \%$ & $42 \%$ & $43 \%$ & $0 \%$ \\
\hline C. ACADEMIC CONSULTATION & $2 \%$ & $15 \%$ & $43 \%$ & $37 \%$ & $3 \%$ \\
\hline $\begin{array}{l}\text { D. TUTORIAL SERVICES } \\
\text { E. PRACTICUM / LAB./ STUDIO }\end{array}$ & $1 \%$ & $10 \%$ & $42 \%$ & $46 \%$ & $1 \%$ \\
\hline SERVICES & $1 \%$ & $11 \%$ & $41 \%$ & $29 \%$ & $19 \%$ \\
\hline F. TEACHING MATERIALS & $1 \%$ & $5 \%$ & $25 \%$ & $36 \%$ & $34 \%$ \\
\hline G. EXAM SERVICE & $2 \%$ & $10 \%$ & $35 \%$ & $54 \%$ & $0 \%$ \\
\hline H. TRANSFER OF CREDIT SERVICES & $3 \%$ & $17 \%$ & $36 \%$ & $21 \%$ & $24 \%$ \\
\hline I. LEARNING EXPERIENCE AT UT & $0 \%$ & $6 \%$ & $37 \%$ & $57 \%$ & $0 \%$ \\
\hline
\end{tabular}

\section{CONCLUSION}

Information on filling out the questionnaire through the WhatsApp application can be conveyed well to students. Generally, student stated FST service is good. Based on this study, it was found that $43 \%$ of students were very satisfied, $38 \%$ said they were satisfied, $10 \%$ said they were quite satisfied, and $1 \%$ felt dissatisfied. 7\% of students did not answer questions, especially in studio practicum services, teaching materials services and credit transfer services. Important information that students want is about General Services, Tutorial Services, Teaching Materials Services, Practicum Services and Exam Services.

The recommendation based on this research is that all Programs at FST develop academic guidance services through WhatsApp group so that students can access information related to public services, tutorial services, teaching materials services, practicum services and exam services. On the other hand, special provisions regarding practicum, the program studies can convey information periodically and using a variety of communication channels, such as WhatsApp Blast, Information through UPBJJ, and FST social media.

\section{REFERENCES}

[1] D. L. Reising, P. N. Allen and S. G. Hall. Student and community outcomes in service-learning: Part 1Student perceptions. Journal of Nursing Education, 45 (12) (2006) 512-515. DOI: https://doi.org/10.3928/0148 4834-20061201-07

[2] A. S Burke and M. D. Bush. Service learning and criminal justice: An exploratory study of student perceptions. Educational Review, 65 (1) (2013) 56-69. DOI: https://doi.org/10.1080/00131911.2011.638138
[3] O. A. Lateef, M. F. Khamidi and A. Idrus. Building maintenance management in a Malaysian university campus: a case study. Construction Economics and Building, 10 (1-2) (2010) 76-89. DOI: https://doi.org/ 10.5130/AJCEB.v10i1-2.1593

[4] P. S. Aithal and S. Aithal. Building World-Class Universities: Some Insights \& Predictions. Building World-Class Universities: Some Insights \& Predictions. International Journal of Management, Technology, and Social Sciences, 4 (2) (2019) 13-35. https://ssrn.com/ abstract $=3443047$

[5] A. Y. C. Hou, R. Morse and C. L. Chiang. An analysis of mobility in global rankings: making institutional strategic plans and positioning for building world-class universities. Higher Education Research \& Development, 31 (6) (2012) 841-857. DOI: https://doi. org /10.1080/07294360.2012.662631

[6] J. Song. Creating world-class universities in China: Strategies and impacts at a renowned research university. Higher Education, 75 (4) (2018) 729-742. DOI: https://doi.org/10.1007/s10734-017-0167-4

[7] R. A. Rhoads, S. Li and L. Ilano. The Global Quest to Build World-Class Universities: Toward a Social Justice Agenda. New Directions for Higher Education, 2014 (168) (2014) 27-39. DOI: https://doi.org/10.1007/ s10734-017-0167-4

[8] R. A. Rhoads, S. Li and L. Ilano. The Global Quest to Build World-Class Universities: Toward a Social Justice Agenda. New Directions for Higher Education, 2014 (168) (2014) 27-39. DOI: https://doi.org/10.1007/ s10734-017-0167-4 
[9] H. Zhang, D. Patton and M. Kenney. Building global-class universities: Assessing the impact of the 985 Project. Research Policy, 42 (3) (2013) 765-775. DOI: https://doi.org/10.1016/j.respol.2012.10.003

[10] J. Lee. Creating world-class universities: Implications for developing countries. Prospects, 43 (2) (2013) 233-249. DOI: https://doi.org/10.1007/s11125013-9266-x

[11] J. L. Ortega and I. F. Aguillo. Mapping world-class universities on the web. Information Processing \& Management, 45 (2) (2009) 272-279. DOI: https://doi. org/10.1016/j.ipm.2008.10.001

[12] J. Li. World-class higher education and the emerging Chinese model of the university. Prospects, 42 (3) (2012) 319-339. DOI: https://doi.org/ 10.1007/ s11125-012-9241-y 